\title{
Desenvolvimento de um Medidor de Consumo de Energia Elétrica em Tempo Real por meio de Medidor não Invasivo de Corrente Utilizando a Plataforma Arduino
}

\author{
Jader Fernndo Dias Breda* Jacilene Martins Medeiros ** \\ João Vitor Gomes de Araújo*** \\ *e-mail: jader.breda@ufvjm.edu.br \\ ** e-mail: jacilene.martins@ufvjm.edu.br \\ *** e-mail: joao.araujo@ufvjm.edu.br \\ Instituto de Engenharia, Ciência e Tecnologia, Universidade Federal \\ dos Vales do Jequitinhonha e Mucuri, Janaúba, $M G$
}

\begin{abstract}
Electricity is one of the most worrying topics today. With the water crisis and a large population growth, the main sources of energy are not enough to supply all the energy demand. Amidst these factors, the price of Kwh becomes higher each year. This consequence results in a great challenge for consumers, which is the control linked to the economy of electricity. Faced with this, researchers and technology lovers have created a practical device for consumers to monitor energy consumption. The objective of this work is to develop the prototype of an energy consumption meter in real time through the measurement of electric current. For development, we used the Proteus integrated circuit design program and the Arduino electronic prototyping platform. With the prototype, it was possible to illustrate in a display: the circuit current, the duration of measurement, the energy consumed over time and the corresponding value in Reais, taking into account the cost of electric energy of a local power company.

Resumo: A energia elétrica é um dos temas mais preocupantes da atualidade. Com a crise hídrica e um grande crescimento populacional, as principais fontes de energia não são suficientes para suprir toda a demanda energética. Em meio a esses fatores, o preço do Kwh torna-se maior a cada ano. Essa consequência resulta em um grande desafio para os consumidores, que é o controle atrelado a economia da energia elétrica. Diante disso, pesquisadores e amantes da tecnologia criaram um dispositivo prático para que os consumidores possam monitorar o consumo da energia. O objetivo desse trabalho é desenvolver o protótipo de um medidor de consumo de energia em tempo real por meio da medição de corrente elétrica. Para o desenvolvimento, foi utilizado o programa de projeto de circuitos integrados Proteus e a plataforma de prototipagem eletrônica Arduino. Com o protótipo foi possível ilustrar em um display: a corrente do circuito, a duração de medição, a energia consumida ao longo do tempo e o valor correspondente em reais, levando em consideração o custo de energia elétrica de uma concessionária de energia local.
\end{abstract}

Keywords: Prototype; Electric Power; Power Consumption; Arduino Platform; Proteus. Palavras-chaves: Protótipo; Energia elétrica, Consumo de energia; Plataforma Arduino; Proteus.

\section{INTRODUÇÃO}

Em aspectos gerais a energia é definida como a capacidade de produzir trabalho e em consequência transmitir calor (Farias and Sellitto, 2011). Pela primeira lei da termodinâmica, a energia interna do sistema é dada pela soma do calor trocado por um sistema e do trabalho realizado por ele ou sobre o mesmo (Zemansky and Dittman, 1981).

$\mathrm{Na}$ física, a energia mecânica é dada pela soma entre, a energia cinética, associada ao estado do movimento de um objeto, e a energia potencial, pela qual está associada ao estado de separação entre dois objetos que se atraem mutuamente devido a força gravitacional (Halliday et al., 2012). A mesma pode ser transformada e transferida de um objeto para outro, ocorrendo no final, a conservação da sua quantidade e o momento linear (Tolmasquim, 2012).

A energia possui uma grandeza escalar associada ao estado de um ou mais objetos (Halliday et al., 2012), no entanto pode se manisfestar de formas diferentes, podendo ser divididas em: energia térmica, luminosa, química, mecânica, atômica, elétrica, entre outras (Melo, 2019).

Em especial a energia elétrica pode ser compreendida como a potência consumida por alguma carga em um determinado período de tempo (Morales, 2007). A sua descrição matemática é dada pela equação (1), onde $\mathrm{P}$ é a potência definida pela quantidade de energia elétrica solicitada na unidade de tempo e expressa em quilowatts 
(kW) (DPP-DNIT, 2009), t é o tempo dado em horas e E é a energia dada em kwh, kJ ou kcal, (Nogueira, 2001). No consumo de eletricidade a unidade usual padrão é o quilowatt-hora (1 kwh= $860 \mathrm{kcal})$ (Ornellas, 2006).

$$
E=P t
$$

O comportamento de rede de distribuição da energia elétrica pode ser vista como um arranjo de muitos pequenos e simples circuitos elétricos (zonas de rede) que possuem soluções lineares obtidas pelas leis de Kirchhoff das correntes e tensões (Leite, 2015).

O consumo médio é a quantidade de energia consumida (kWh) pelos clientes, dividida pelo número de clientes (DPP-DNIT, 2009). Com uma taxa de crescimento anual média de $7,35 \%$, o consumo total de energia elétrica passou de 18.346 GWh em 1960 para 304.634 GWh em 2000 (Schmidt and Lima, 2004). Foi estimado que em 2020 o consumo de eletricidade será $61 \%$ superior ao ano de 2010, podendo resultar em 730 TWh (Tolmasquim, 2012).

As necessidades energéticas do homem estão em constante evolução (Farias and Sellitto, 2011). Antes era inimaginável que as reservas ambientais poderiam um dia serem limitadas (Petry et al., 2010). A escassez de recurso em consequente aumento do custo de energia elétrica foram determinantes para a adoção de ações de gerenciamento energético pelas empresas (Cunha, 2010). Como a necessidade de reduzir o consumo de energia, sem causar uma desarmonia na economia do país (Schmidt and Lima, 2004).

A economia de energia é um tema bastante discutido por todos, principlamente dentro de comitês estabelecidos pelas empresas. Esse assunto tem ganhado grandes destaques e o fator econômico, o uso racional e eficiente da energia se torna um desafio constante para toda a sociedade (Metrum, 2019)

A Agência Nacional de Energia Elétrica (ANEEL) têm implementado o sistema de bandeiras tarifárias, com o objetivo dos seus consumidores terem uma noção aproximada do valor da tarifa de energia dos próximos meses, de acordo com suas condições de uso (ANEEL, 2008).

Em busca de implementar medidas energéticas mais eficazes, otimizar investimentos, evitar custos adicionais nas contas de energia e se beneficiar de menores tarifas, muitos pesquisadores e organizações têm procurado novas tecnologias que as auxiliem no gerenciamento da energia elétrica (ATK, 2016).

Sistemas de gerenciamento de energia são sistemas de automação que coletam dados de medição de energia do campo e os disponibilizam aos usuários, por meio de relatórios, gráficos, ferramentas de monitoração online e analisadores de eventos de qualidade de energia, permitindo assim a gestão de recursos energéticos de uma planta industrial (Alves et al., 2011).

Os sistemas de medição e gerenciamento de energia possibilitam que o cliente tenha conhecimento e controle do seu consumo de energia, podendo acarretar na diminuição de gastos desnecessário (Electric, 2019). Para análise e gerenciamento dos recursos energéticos é importante que as informações sejam dispostas de forma que haja uma alta confiabilidade (CCK, 2019).

Atualmente, o meio mais comum de transmissão de dados é conectando as subestações aos medidores instalados ao computador ou servidor em que os dados são processados e armazenados (Alves et al., 2011). Ter um sistema de monitoramento de energia elétrica é sem dúvida uma vantagem econômica, competitiva e estratégica para qualquer indústria (Kremen, 2016).

Sendo assim, o objetivo deste é desenvolver um medidor de consumo de energia elétrica por meio da medição de corrente elétrica utilizando a plataforma de prototipagem eletrônica Arduino, para que a partir deste protótipo o consumidor possa saber em tempo real o valor que está consumindo e pagando durante o período de medição.

\section{METODOLOGIA}

O Arduino é uma plataforma de prototipagem de hardware livre, composta por um microprocessador Atmel capaz de gerar dados de entrada e saída, facilmente conectada a um computador. A sua programação é via IDE (Integrated Development Environment, ou Ambiente de Desenvolvimento Integrado), na qual suporta a linguagem de programação $\mathrm{C} / \mathrm{C}++$. Essa plataforma é flexível, de baixo custo e fácil de programar, em que por tais características foi escolhido para o desenvolvimento do protótipo.

Inicialmente foi desenvolvido o código de programação no IDE da plataforma Arduino, onde o mesmo recebe o valor da corrente originada pela distribuição da concessionária de energia elétrica local, pela qual o seu valor é visto em um display. Para o protótipo foi usado o display Nokia 5110 , por ser o mais popular.

No IDE do Arduino foi utilizado as bibliotecas Adafruit GFX, Adafruit PCD e EmonLib. A biblioteca GFX proporciona várias funções gráficas para todos os LED e Displays, na qual permite que os esboços do Arduino sejam facilmente adaptados (Burges, 2018). A PCD é uma biblioteca própria para o monitor LCD monocromático Nokia 5110, que usa a comunicação SPI (Serial Peripheral Interface) para se comunicar, onde são necessários 4 ou 5 pinos para interface (Burges, 2012). A SPI é um protocolo de dados seriais síncronos utilizado em microcontroladores para comunicação entre o microcontrolador e um ou mais periféricos (ARDUINO, 2019). E para receber os dados do sensor de corrente, foi utilizado a biblioteca EmonLib.

No código foi calculado a potência, energia e o custo da energia em kWh. A fim de prevenir riscos, como o de queimar o equipamento, utilizou-se o programa de projeto de circuitos integrados Proteus. Em que foi possível criar e simular o circuito elétrico necessário para o medidor de consumo de energia elétrica, usando o display Nokia 3310.

Posteriormente, mediante o seu bom funcionamento no Proteus foi desenvolvido a parte física do protótipo. A princípio foi testado o funcionamento do display com o auxílio de uma protoboard, jumpers e um Arduino Uno. Este é o mais utilizado no mercado uma vez que possui uma ótima quantidade de portas disponíveis e grande compatibilidade com os Shields, que são placas de circuitos capazes de aumentar a sua capacidade, como a inserção de 
displays e sensores. Para esse trabalho foi observado que o Uno possui mais informações na literatura, a título de consultas, além de possuir várias bibliotecas prontas. Por ser um protótipo o tamanho da plataforma Arduino pode ser desprezado.

Com a adição do sensor de corrente sensor de corrente SCT-013-000 de 100 A, foi possível analisar os valores dos dados de saída do display. Por fim como o arduino suporta apenas valores positivos foi criado o circuito polarizador divisor de tensão, composto por dois resistores, $R_{1}$ e $R_{2}$, onde se o divisor for bem projetado torna o transistor muito mais estável. A ideia básica é dividir a tensão VCC em dois valores estáveis de modo que a tensão de $R_{2}$ seja constante.

Com o desenvolvimento do código de programação, o bom funcionamento do display e a adição do circuito polarizador, foi possivel a criação do protótipo do medidor de consumo, onde foram obtidos os valores da corrente, energia elétrica e o custo por kWh.

\section{RESULTADOS E DISCUSSÃO}

A priori o medidor de consumo de energia foi desenvolvido no programa de projeto de circuitos integrados Proteus. A Figura 1 representa a plataforma Arduino, que funciona como um canal de ligação entre o código de programação e o display. Essa figura mostra todas as pinagens do Arduino em especial o VCC que corresponde a uma diferença de potencial e representa o nível lógico binário do Arduino (0 e 1$)$, variando suas voltagens de $0 \mathrm{~V}(\mathrm{LOW}=0)$ até $5 \mathrm{~V}$ $(\mathrm{HIGH}=1)$.

Na Figura 2 tem-se o display Nokia 3310, composto por 7 pinos capazes de se conectarem ao Arduino para a exibição de dados de saída. O pino GND pode ser considerado como a referência do VCC, podendo ser o mesmo que o terra, para o funcionamento do display 3310 foi adicionado um resistor $220 \omega$ ao pino do LED com o VCC. O pino RST é o Reset, o CLK é do relógio (clock), DIN é a linha da data, DC data/instrução de seleção e o CS está relacionado com a habilitação do chip, todos conestados a uma pinagem específica do Arduino, como mostra a figura.

Um medidor de corrente é interligado ao pino $A_{1}$ do Arduino, mas por ser analógico é impossibilitado de fazer a leitura de corrente, para tal deve ser adicionado um circuito que converte as leituras em tensão. Como mostra o circuito da Figura 2, ao colocar um resistor de carga indutiva $R_{7}$ paralelo a bobina do medidor de corrente (isine), é obtido uma tensão no circuito. Para obter o valor do resistor $R_{7}$, deve ser analizado as correntes de pico de entrada e saída do medidor de corrente. Como foi usado um medidor de $100 \mathrm{~A}$, logo a corrente de pico de entrada é dada pela equação 2 .

$$
I_{1}=I_{m} I_{e f}=100 \sqrt{2}=141,42 \mathrm{~A}
$$

Onde Im é a corrente pré-fixada do medidor de corrente e Ief é a corrente eficaz, isto é, um cálculo médio estatístico de um valor variável. A corrente da bobina secundária do sensor é mostrada na equação (3).

$$
I_{2}=\frac{I_{1}}{\text { voltas }}=\frac{141,42}{2000}=0,07 \mathrm{~A}
$$

Em que voltas são as quantidades de voltas do fio na espira do medidor de corrente, que pode ser dada pela equação (4):

$$
\frac{N_{1}}{N}=\frac{I_{f}}{I_{i}}
$$

Em que $N_{1}$ é 1 volta, $I_{i}$ é a corrente inicial de $100 \mathrm{~A}$ e $I_{f}$ é a corrente final produzida por ela que é $50 \mathrm{~mA}$ ou 0,05 A. Dessa forma, $N_{2}=2000$ voltas. Mas pela lei de Ohm a tensão é mostrada na equação (5).

$$
v=R_{i}
$$

Sabendo que o sinal de onda senoidal resultante varia de 0 a 5 , ou de $-2,5$ a 2,5 , se contar os valores negativos, considerando então, uma tensão de $2,5 \mathrm{~V}$ e uma corrente sencundária de $0,07 \mathrm{~V}$. Tem-se uma resistência de carga igual a $35,7 \Omega$, no entanto, como não foi possivel obter esse valor de resistência, foi utilizado um resistor de $33 \Omega$, como mostra a Figura 2.

Mas como a plaforma Arduino não consegue ler valores negativos, foi usado o polarizador divisor de tensão na base, com o intuito de elevar a onda senoidal de $-2,5 \mathrm{~V}$ para $+2,5 \mathrm{~V}$, isto é, para o polo positivo acima do potencial da terra para um transistor, que no caso do protótipo está contido no display. O pino da luz de fundo desse é conectado a um transistor PWM para que se possa acender todos os 4 LEDs do display ao mesmo tempo a partir de um pino do microcontrolador.

O transistor é um componente ativo de um circuito amplificador de tensão ou corrente. Eles são como uma válvula, composta por ums entrada (coletor), uma saída (emissor) e uma entrada de controle (base). Polarizar um transistor consiste em ligá-lo de forma que uma corrente circule na direção junção Base-Emissor e na direção junção ColetorEmissor.

Como o polarizador escolhido foi o divisor de tensão na base, logo foi adicionado ao circuito 2 resistores divisores de tensão, $R_{5}$ e $R_{6}$, ambos de $10 \mathrm{~K} \Omega$. Esse divisor resulta em um potencial $\mathrm{V}$ no terminal base do transistor que polariza diretamente a junção base-emissor, produzindo assim a corrente de base quiescente I. Para que não ocorresse a variação da tensão, vou adicionado um capacitor ao circuito. Posteriormente foi inserido o código de programação ao Proteus, onde foi possivel simular e analisar os dados obtidos.

A posteriori, diante do seu bom funcionamento no software simulado, foi desenvolvido a parte física do medidor de corrente. A princípio montou-se o circuito que interliga o arduino com o display, como pode ser observado na Figura 3 . Nele, foi testado o tamanho e posição dos dados de saída.

No código de programação arduino por meio da biblioteca Adafruit, as configurações de saída foram dadas por:

display . begin();

display. setContrast (55); 


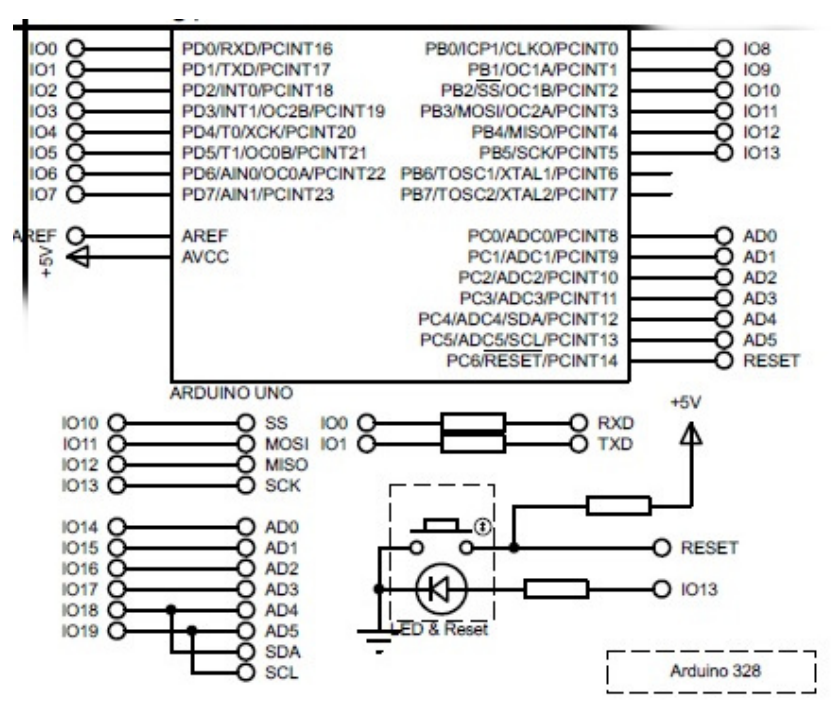

Figura 1. Circuito do Arduino no Proteus.

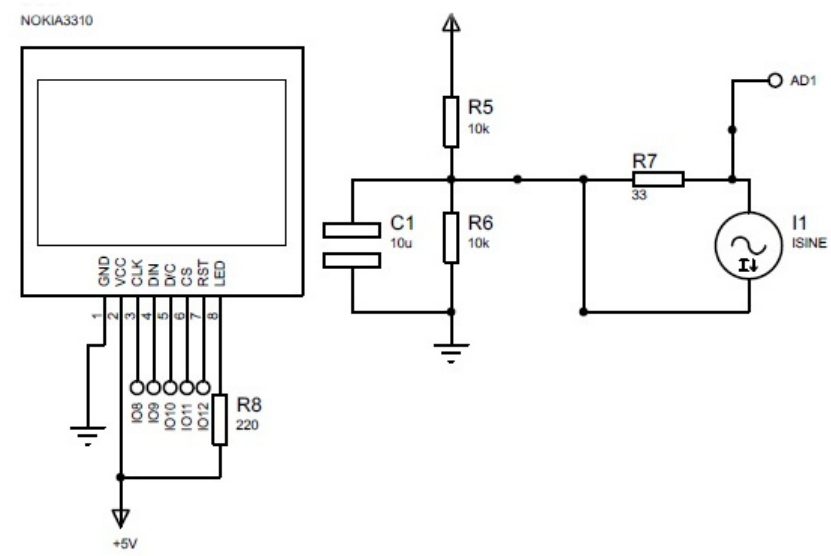

Figura 2. Circuito do Arduino no Proteus.

$$
\begin{aligned}
& \text { display.clearDisplay(); } \\
& \text { display.setTextSize(0); } \\
& \text { display.setTextColor (BLACK); } \\
& \text { display.drawRect }(0,0,84,48,2) \text {; }
\end{aligned}
$$

Pelo qual, o comando begin faz os ajustes iniciais do display, o comando setCrontrast define o contraste do display (o 55 foi um valor de teste), o comando clear apaga o buffer do display, o setTextSize e o color, determinam a fonte e a cor do texto, respectivamente e por fim, o comando drawRect cria uma borda retangular no display.

Para aparecer no display o nome dado para uma certa gradeza medida ou calculada, como por exemplo a energia consumida $(\mathrm{E}(\mathrm{kwh}))$. No setup da programação IDE do arduino foi inserido o código:

display.setCursor $(2,25)$;

display.println("E(kwh):");

Em que, no comando setCursor, o 2 é a posição da coluna e o 25 é a posição da linha. E para aparecer os dados de saída no loop do arduino foi inserido o código:

display.setCursor $(51,25)$;

display.fillRect $(50,25,33,8,0)$;

display.println (energkwh);
Em que o setCursor analisa a posição e a linha do valor e pelo fato de ter tido uma valor de oscilação, o fillRect apaga o valor anterior da tela.

Assim, montou-se o circuito polarizador, como mostra a Figura 4 Onde a corrente que vem do circuito que está sendo medido passa por esse circuito polarizador, que de certa forma ele desloca a parte negativa da senoide para a parte positiva, devido a porta de entrada do arduino medir somente a parte positiva.

Através do circuito polarizador montado o sensor de corrente SCT-013-000 de 100 A não invasivo ligado ao circuito que se desejava ser analisado, foi feito as medições das correntes que passava pelo circuito polarizador, com isso foi possível verificar corretamente os valores de saída que o sensor estava medindo.

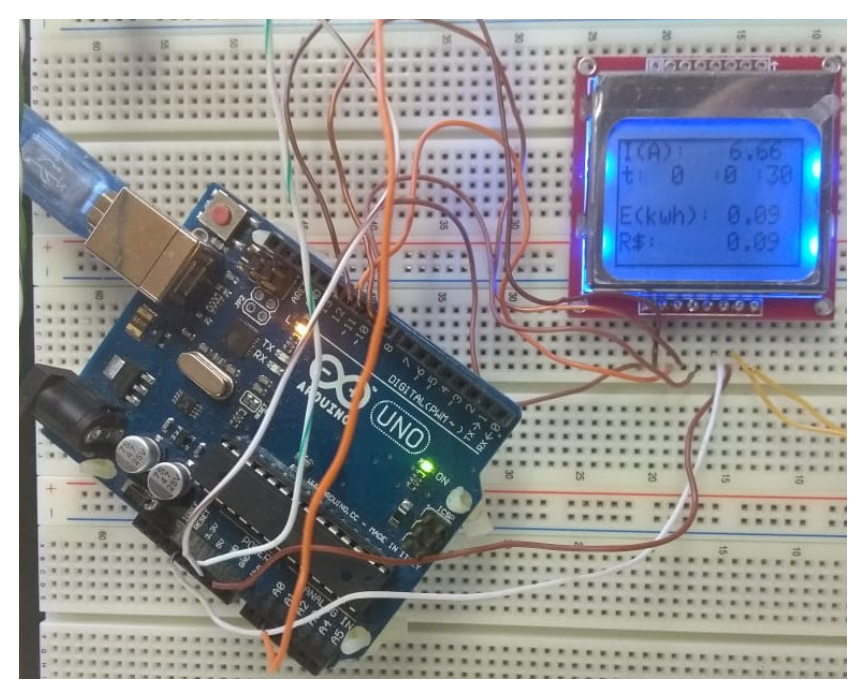

Figura 3. Circuito Arduino-Display.

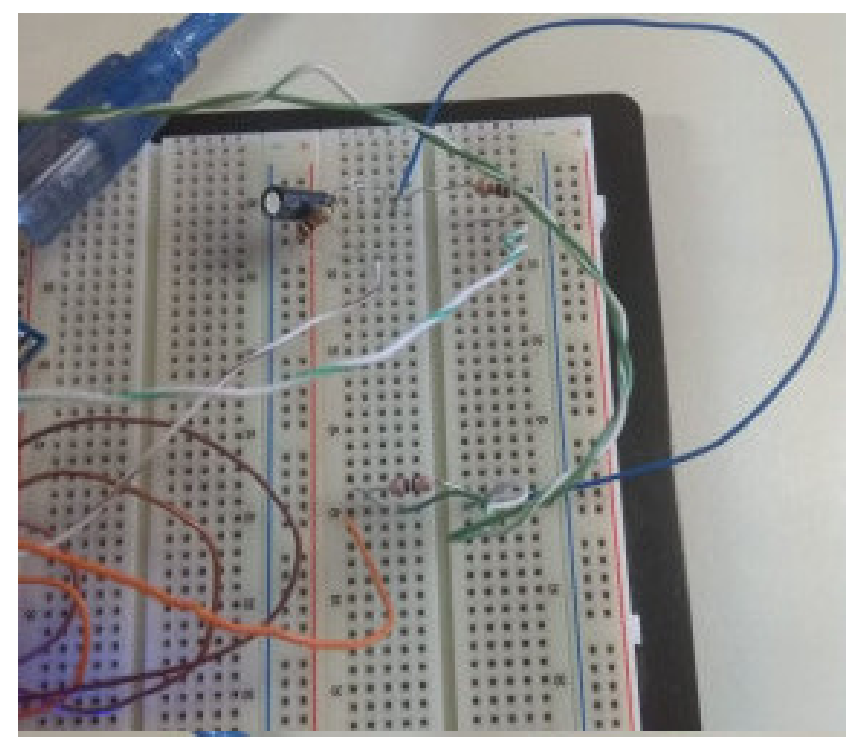

Figura 4. Circuito do Arduino no Proteus.

Logo, através de cada parte do circuito montada corretamente, foi tudo integrado a um circuito completo, com isso foi possível carregar um programa apropriado no arduino, onde através desse programa foi observado que no display Nokia 5110 estava exibindo os valores que eram desejados, 
como a corrente (A), o tempo (t) no formato de um cronômetro (h:m:s), a energia (kWh) e o custo $(\mathrm{R} \$)$, tudo em tempo real.

Após, o funcionamento do medidor de consumo de energia foi testado em um circuto elétrico do pavilhão de aulas da UFVJM. Como pode ser visto na Figura 5, por ser não invasivo, o sensor de corrente foi inserido em uma das duas fiações da instalação elétrica.

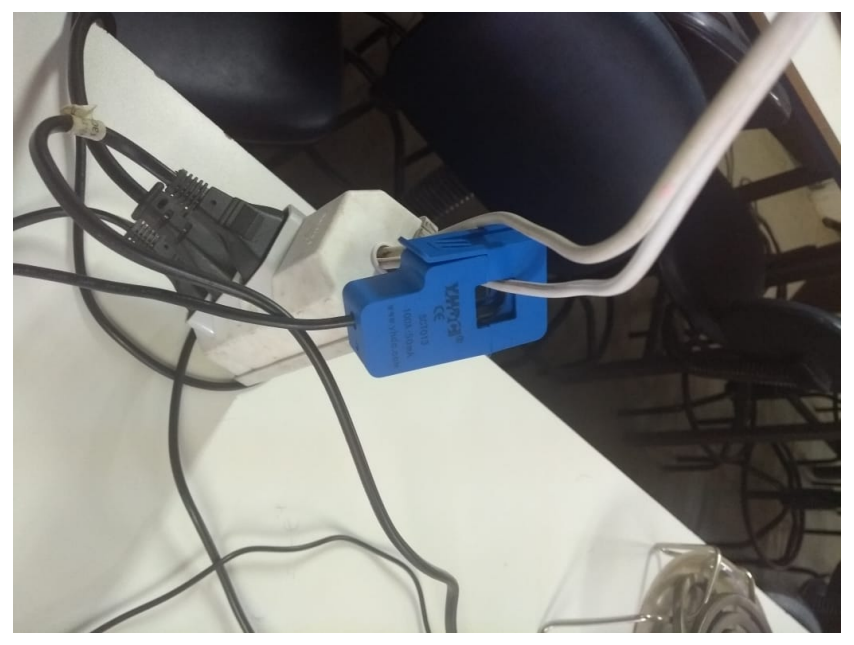

Figura 5. Sensor de Corrente SCT-013-000 não invasivo.

As fiações contém acúmulos de cargas positivas e negativas, onde pela lei de Ampère, gera um campo magnético. Pela lei de Faraday, o campo magnético gerado, induz uma corrente elétrica variável (alternada) na espira contida no interior do sensor de corrente. Essa corrente que segue para o circuito polarizador. Onde, por intermédio do circuito integrado como mostrado na Figura 5 gera os dados de saída, mostados na Figura 6.

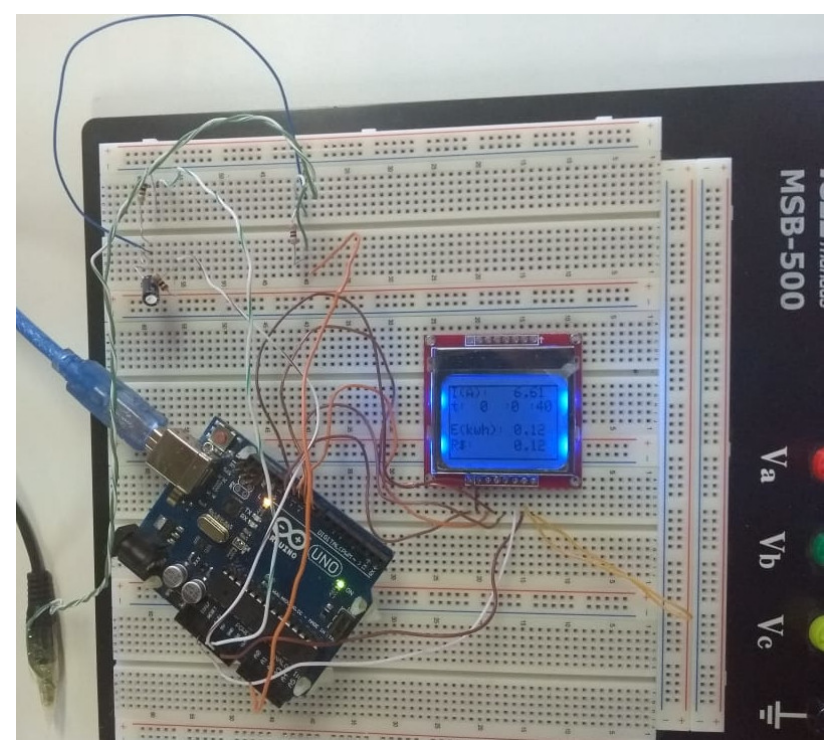

Figura 6. Circuito real de medidor de corrente.

Como mostrado na Figura 6, o primeiro dado a ser obtido é o valor da corrente elétrica, recebida no código de programação do arduino, pelo sensor de corrente. Para tal, foi inserido a função da biblioteca EmonLib no código:

double Irms = emon1. $\operatorname{calcIrms}(1480)$;
Com o valor da corrente, foi calculado a potência, dada pela expressão:

$$
P=i . v
$$

Onde $\mathrm{P}$ é a potência, i é a corrente e v é a tensão, em que essa foi definida como constante $(220 \mathrm{~V})$, considerando que não deve haver uma variação significativa na tensão fornecida pela concessionária de energia. Foi definido também o tempo (t) de medição, dado por um cronômetro, que mostra as horas, os minutos e os segundos. Assim, com o valor da potência e do tempo, foi calculado o valor da energia elétrica gasta:

$$
E=P . t
$$

Energia dada em kWh. Assim, considerando que a energia elétrica fornecida pela concessionária de energia local é $\mathrm{R} \$ 0,95$ o $\mathrm{kWh}$, assim, o custo da energia elétrica é dado por:

$$
\text { Custo }=\text { E.(0,95) }
$$

No código, a fim de diminuir os erros de medição, foi analisado as potências e energias médias de várias amostras. Tornando-o:

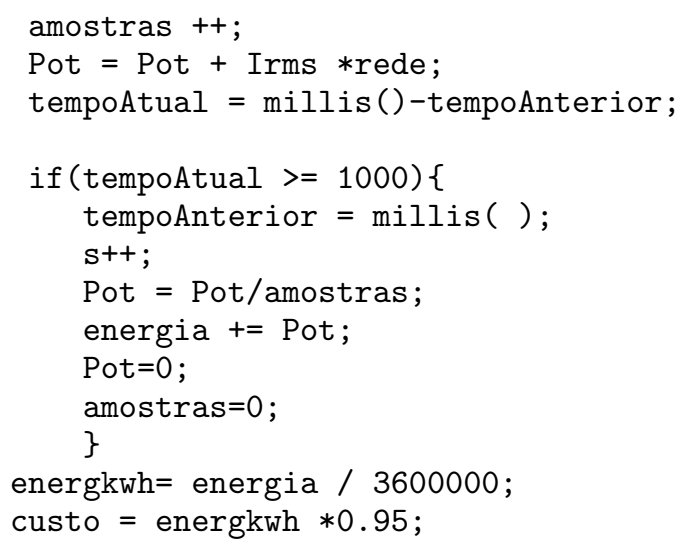

Assim, a cada intervalo de tempo transcorrido, vários valores vão sendo acumulados, onde posteriormente são calculados os valores de potência e energia elétrica média.

Como pode ser visto no display, o tempo é marcado no formato de cronômetro. Para tal, no if temporal foi adicionado o código:

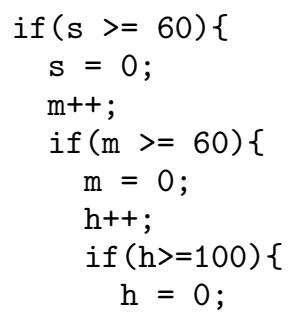

Onde s representa os segundos, m os minutos e h as horas.

\section{CONCLUSÃO}

Nesse trabalho foi desenvolvido um protótipo de um medidor de consumo de energia elétrica utilizando a plataforma 


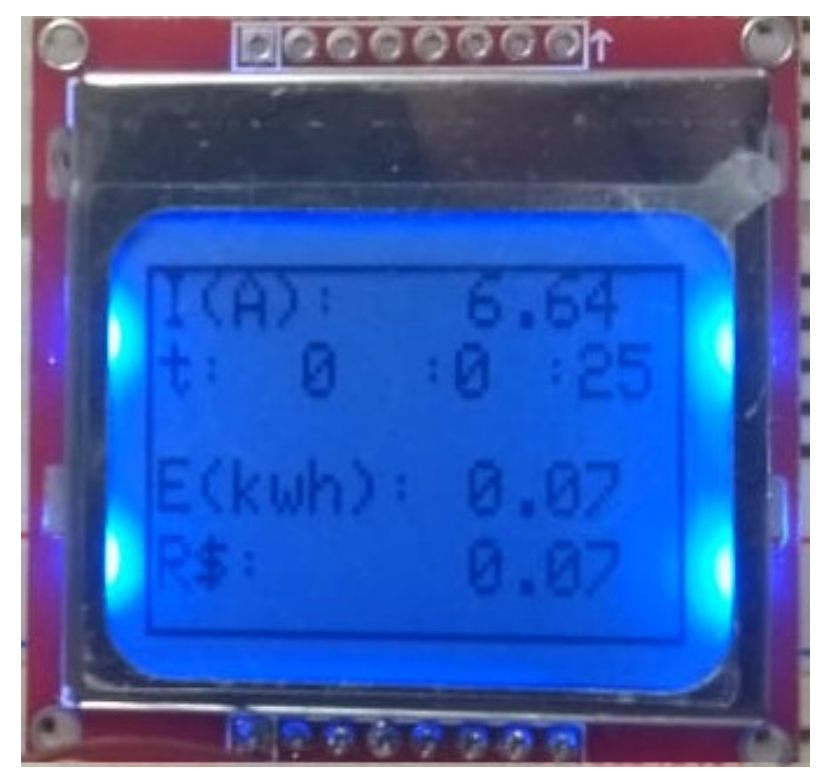

Figura 7. Valores medidos em tempo real no Display Nokia 5110.

de prototipagem Arduino, para tal foi escolhido um sensor de corrente do tipo não invasivo, que não apresenta a necessidade de abrir todo um circuito para se medir a corrente. O mesmo teve um funcionamento adequado, detro do esperado, onde foi possivel medir a corrente, e através das medições da corrente, foi obtido os valores de potência e energia na qual teve o mínimo de variações, devido o circuito polarizador utilizado.

Por meio de toda essa estrutura construida, os valores medidos foram mostrados em tempo real no display, juntamente com o tempo desde que o sistema foi ligado. E ao calcular o valor da tensão usando os dados obtidos no display, foi comprovado que o sensor junto com o arduino fez uma leitura correta, obtendo $220 \mathrm{~V}$. Sendo assim, pode ser concluído que todos os objetivos foram alcançados e obtiveram resultados satisfatórios.

Para trabalhos futuros, o medidor de corrente poderá ser compactado em um produto portátil, a fim de ser utilizado em aulas práticas e/ou para comercialização. Além disso, o produto pode ser extendido com a inserção de mais medidores de corrente. E para garantir ainda uma melhor precisão, medidores de tensão também podem ser acoplados ao equipamento.

\section{AGRADECIMENTOS}

Os autores gostariam de agradecer ao Instituto de Engenharia, Ciência e Tecnologia da Universidade Federal dos Vales do Jequitinhonha e Mucuri-Campus Janaúba, pela estrutura disponibilizada e por todo apoio prestado para a realização deste trabalho.

\section{REFERÊNCIAS}

Alves, D., Ribeiro, D., Santos, L., Camilo, J.C., and Anjos, R. (2011). Características e benefícios dos sistemas de gerenciamento de energia. 150-161.

ANEEL (2008). Por dentro da conta de luz. Brasília.

ARDUINO (2019). Uma breve introdução à interface periférica serial (spi). Disponivel em:
https://www.arduino.cc/en/reference/SPI. /acessado em 2019.

ATK (2016). A importância de um sistema de gerenciamento de energia elétrica e utilidades. Disponivel em: http://atk.net.br/blog/2016/01/gerenciamentode-energia-eletrica/. Acessado em 2019.

Burges, P. (2012). Por dentro da conta de luz. Nova York. Burges, P. (2018). Por dentro da conta de luz. Nova York. CCK (2019). Gerenciamento de energia. Disponível em: www.cck.com.br/artigos/gerenciamento.php. /acessado em 2019.

Cunha, L. (2010). Gerenciamento de energia no brasil. Portal o setor da energia elétrica.

DPP-DNIT (2009). Cálculo de Demanda para Medição de Cliente em Baixa Tensão. Rio de Janeiro.

Electric, S. (2019). Sistemas de medição e gerenciamento de energia. Disponível em: http://201.55.65.27/sistemas-de-medicao-egerenciamento-de-energia/. Acessado em 2019.

Farias, L.M. and Sellitto, M.A. (2011). Uso da energia ao longo da história: evolução e perspectivas futuras. Revista Liberato, 12(17), 01-106.

Halliday, D., Resnick, R., and Walker, J. (2012). Fundamentos de física, vol. 1: mecânica. LTC. $g^{a}$.

Kremen, K.d.S. (2016). O gerenciamento de energia elétrica fábril. Revista Technoeng, 1(13), 269-279.

Leite, J.B. (2015). Desenvolvimento de um sistema de gerenciamento de energia (ems-energy management system) para a rede elétrica inteligente (smart grid).

Melo, A. (2019). Física. energia. Disponível em: https://docplayer.com.br/82805284-Fisica-energiamecanica-augusto. Acessado em 2019.

Metrum (2019). Sistema integrado de gerenciamento de energia (sige). Disponivel em: http://memt.com.br/sistema-de-gerenciamento-deenergia/. Acessado em 2019.

Morales, C. (2007). Indicadores de consumo de energia elétrica como ferramentas de apoio a gestão: classificação por prioridades de atuação na universidade de são paulo. Universidade de São Paulo.

Nogueira, L. (2001). Energia: conceitos e fundamentos. Conservação de Energia eficiência energética de instalações e equipamentos. Itajubá: Editora da EFEI.

Ornellas, A.J. (2006). A energia dos tempos antigos aos dias atuais. Universidade Federal de Alagoas. Maceió: EDUFAL.

Petry, B.M., da Silva, A.K., Moreira, D.R., and Ramon, G. (2010). Projeto use-uso sustentável da energia na pucrs. Anais do XI Salão de Iniciação Científica.

Schmidt, C.A.J. and Lima, M.A. (2004). A demanda por energia elétrica no brasil. Revista brasileira de economia, 58(1), 68-98.

Tolmasquim, M.T. (2012). Perspectivas e planejamento do setor energético no brasil. Estudos avançados, 26(74), $247-260$.

Zemansky, M. and Dittman, R. (1981). Heat and Thermodynamics. McGraw-Hill Book Co. 